\title{
Rapid method for fetal brain fixation
}

\author{
J M NICHOLLS Department of Histopathology, The Queen Elizabeth Hospital, Woodville, South Australia
}

SUMMARY A quicker alternative to the standard removal and fixation of brain tissue was sought. Whole fetal brains were fixed in situ using a mercuric based fixative. The subarachnoid space was perfused overnight with Heidenhain's Susa fixative. The following day the brains were removed from the cranium in the standard manner. After storage for three days in Susa's fixative the brain was sliced and processed, with excellent preservation of gross and microscopic architecture. The cost is only marginally higher than that of commercial formalin.

Fetal brains are soft and often damaged when removed from the cranium. In cases of maceration the brain is semi-liquid and cannot be removed intact. Various methods have been described to facilitate removal and fixation of the brain ${ }^{1}$ but these require fixation for periods of one to six weeks. Traditionally, the most common fixative has been $10 \%$ neutral buffered formalin with concentrated $(40 \%$ formaldehyde) formalin solution used occasionally. ${ }^{1}$ The method described below has been used in over 20 fetal brains and uses a mercuric based fixative. It has two advantages: the architecture is preserved and the brain can be sectioned after three days' fixation.

\section{Material and methods}

The scalp is reflected in the standard manner. ${ }^{2}$ Once the anterior and posterior fontanelles are exposed a 1 to $2 \mathrm{~mm}$ nick is made with a No 15 scalpel blade in the anterior fontanelle 2 to $3 \mathrm{~mm}$ lateral to the midline. Cerebrospinal fluid cultures can be taken at this stage if required. A new or used epidural catheter is inserted into this nick and fed medially and anteriorly until contact with the base of the cranium is reached. The subarachnoid space is then perfused with Heidenhain's Susa fixative. ${ }^{3}$ The volume depends on the gestation of the fetus. Fetuses of 16 weeks' gestation require only $5 \mathrm{ml}$; those of term gestation may require upwards of $40 \mathrm{ml}$. The injection is performed until the fontanelles become taut but not distended. In larger fetuses the posterior fontanelle may be injected in a similar way. The skin is returned to the normal anatomical position and the fetus is left overnight in the mortuary refrigerator.

Accepted for publication 7 April 1988
The following day the skull is opened using a modification of the Beneke technique $e^{\prime}$ or that proposed by Valdes-Dapena and Huff. ${ }^{2}$ It will be noted that the brain is often a bright white colour instead of the usual light tan colour. After removal the brain is weighed and placed in an adequate volume of Susa's fixative. Full term brains may be sectioned once to allow access of the fixative to the ventricles, but it has been found that this is not necessary for brains under 36 weeks' gestation. The brain is stored for three days, washed, and then cut. The procedure may be delayed for up to three weeks, however, if the tissue is left in fixative for longer. The microtome sections may then be difficult to cut, with some chattering artefact of the section. This has proved the only main drawback to the technique. The cut slices can be retained in fixative for further study after the initial blocks have been taken, but yet again, a degree of hardness will be

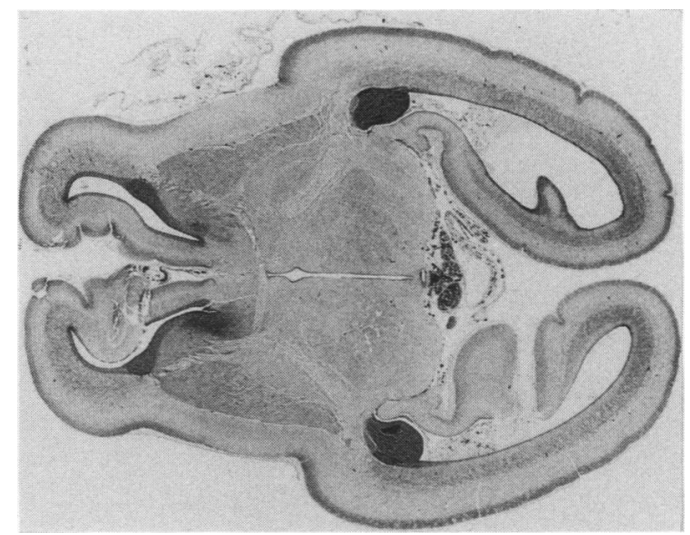

Fig 1 Transverse section of brain at 18 weeks' gestation. (Haematoxylin and eosin.) 


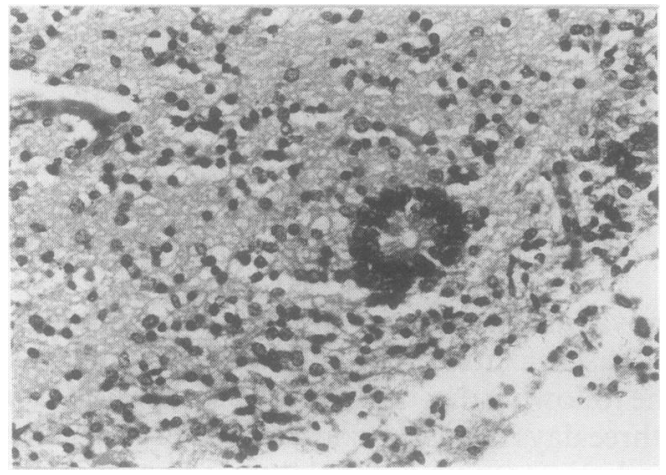

Fig 2 Ependymal differentiation in cerebral neuroblastoma of fetus of 24 weeks' gestation. (Haematoxylin and eosin.)

encountered if sections are taken after three weeks. Mercury will discolour metal knives so running water is essential for frequent rinsing of instruments. Mercury is also a toxic waste so the residual solution has to be stored in a separate container, either for disposal or for future brain fixation. The fixative is marginally more expensive than commercial formalin but this cost is negligible when the amount of fixative used and the reusability of the fixative is considered.

\section{Results}

Microscopic sections are of a high quality with excellent preservation of anatomical detail (figs 1-3). The mercuric pigment is removed using a standard alcoholic-iodine solution and does not prevent the use

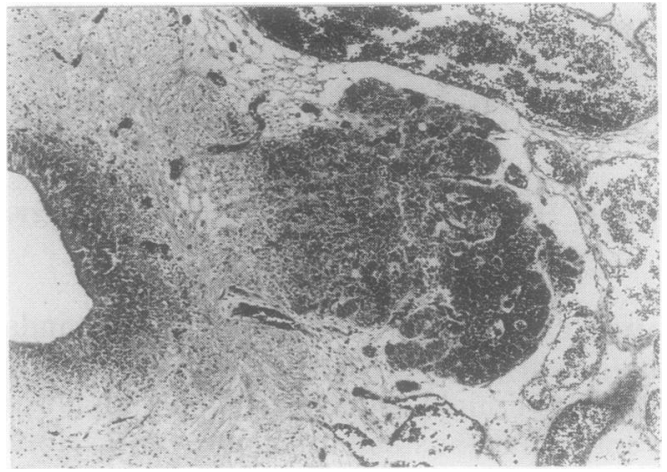

Fig 3 Pineal gland from fetus of 18 weeks' gestation. (Haematoxylin and eosin.)

of immunoperoxidase studies. The brain is firm when cut, permitting accurate assessment of cerebral and cerebellar architecture.

\section{References}

I Wigglesworth JS. Perinatal Pathology. In: Major problems in pathology: Vol 15. New York: WB Saunders Company. 1984.

2 Valdes-Dapena M, Huff D. Perinatal autopsy manual. WashingIon, DC: Armed Forces Institute of Pathology, 1983.

3 Bancroft JD, Stevens A. Theory and practice of histological techniques. 2nd Edition. Edinburgh: Churchill Livingstone. 1982:32.

Requests for reprints to: Dr J M Nicholls, Department of Histopathology, The Queen Elizabeth Hospital, Woodville Road, Woodville, South Australia 5011. Australia. 